\title{
On Some Method for Intrusion Detection Used by the Multi-agent Monitoring System
}

\author{
Agnieszka Prusiewicz \\ Institute of Information Science \& Engineering, Wrocław University of Technology, Poland \\ Wybrzeże Wyspiańskiego 27, 50-370 Wrocław, Poland \\ agnieszka.prusiewicz@pwr.wroc.pl
}

\begin{abstract}
In this paper an original method for intrusion detection used by the multi-agent monitoring system is proposed. Due to the complexity of the problem of the network security maintaining it is a good idea to apply the multiagent approach. The multi-agent system is used in the tasks of computer network monitoring in order to detect the security policy violations. In this paper the algorithm for detecting some type of attack is proposed. This algorithm is based on the network traffic analysis.
\end{abstract}

\section{Introduction}

In this paper an original method for intrusion detection used by the multi-agent monitoring system is proposed. The problem of the network security is taken up since eighties [9] and is developed up today [5, 8, 20]. A comprehensive survey of anomaly detection systems is presented in [16].

A comparison of different approaches to intrusion detection systems is given in [4]. The general challenge of the current intrusion detection systems is to find the difference between the normal and abnormal user behaviour. Intrusion detection system (IDS) should not only recognise the previously knows patterns of attack, but also react in case of appearance of the new events that violate the network security policy. The first models of IDS systems were centralised namely data were collected and analyzed on a single machine. However, the distributed nature of the task of the network security monitoring requires applying of the distributed tools for network security maintaining. Many examples of the distributed IDS systems are given in [1].

The most important postulate addressed to the intrusion detection systems is that, such systems should automatically react in case of detecting the security policy breach to prevent the attack execution or to reduce the potential loss in the network systems. IDS systems should be equipped with the components responsible for the permanent observation of the states of monitored nodes and components that integrate the results of these observations and diagnose the security level of the system. It is reasonable to apply the agency for solving the tasks of the network security monitoring. A multi-agent approach in a network monitoring system was used in works $[7,3$, $10,19]$ where the general system architecture was proposed.

In our approach we also apply the agency to solve the tasks of network security monitoring. In work [11] the framework of an original proposal of the intrusion 
detection system based on the multi-agent approach was presented. In particular, the architecture of such system and the task of agents were specified. Proposed ideas were further developed and in the work [14] the problem of anomalies detection on the basis of the nodes traffic analysis was discussed. Then in the work [17] the agents' knowledge organisation and the general idea of the algorithm for detecting the distributed denial of service $(D D o S)$ attack was proposed. In this work we develop the algorithm for detecting the distributed denial of service attack.

\section{The General Agents' Knowledge Structure}

It is assumed that two types of agents are in the monitoring system: managing agents and monitoring agents. Each monitoring agent $m a$ is responsible for one monitoring region $m r$ consisted of the set of nodes $V$. Managing agents may modify the size of the monitoring regions by adding or removing nodes from the set $V$. It is assumed that the monitoring regions may overlap.

\subsection{The Characteristic of the Monitoring Agents}

The agent $m a$ observes the states of the nodes from his monitoring region $m r$ with a reference to the some properties from the set $P$. $m a$ stores all the observations in his private database $D B$.

Definition 1. A single observation of agent $m a$ is a tuple:

$$
O(v,(p, x), t) \in D B
$$

where $v \in m r, p \in P, t \in T, T$ is the universe of the timestamps and $D B$ denotes the database of the agent $m a$.

Single observation $O(v,(p, x), t)$ refers to the situation that at the timestamp $t$ the agent $m a$ has observed in the node $v$ the value of the parameter $p$ equals $x$ [17].

$D B$ consists of the set of observations (see definition 1) and communication data. Communication data consists of the communication matrix $C M$ of the timestamps in which the communication between the nodes took place.

The second module embodied in the monitoring agent is the anomaly detection module that is supplied by the observations from the set of observations. This module is responsible for data analyzing to detect the security policy violation in the network system [17].

Definition 2. An anomaly detection module of agent $m a$ is defined as a triple:

$$
A D=\left\langle D B_{\left[t_{b^{\prime}}, t_{\left.e^{\prime}\right]}\right.}, A T, A C\right\rangle
$$

where:

- $D B\left[t_{b^{\prime}}, t_{e^{\prime}}\right]$ is a subset of $D B$ restricted to time interval $\left[t_{b^{\prime}}, t_{e^{\prime}}\right]$ and is defined as:

$$
D B\left[t_{b^{\prime}}, t_{e^{\prime}}\right]=\left\{O(v,(p, x), t): p \in P, v \in m r ; t \in\left[t_{b^{\prime}}, t_{e^{\prime}}\right]\right\}
$$


For a chosen time interval $\left[t_{b^{\prime}}, t_{e^{\prime}}\right]$ each tuple $O(v,(p, x), t) \in D B$ that fulfils the following condition: $t \in\left[t_{b^{\prime}}, t_{e^{\prime}}\right]$ is sent into the anomaly detection module $A D$.

- $A T$ is a table of discovered anomalies defined as:

$$
A T=\left\{A\left(v, p, \alpha,\left[t_{b}, t_{e}\right]\right): p \in P, v \in m r ;\left[t_{b}, t_{e}\right] \subseteq\left[t_{b^{\prime}}, t_{e^{\prime}}\right]\right\}
$$

A single anomaly $A\left(v, p, \alpha,\left[t_{b}, t_{e}\right]\right) \in A T$ is interpreted as the agent $m a$ believes at the level $\alpha$ that in the time interval $\left[t_{b}, t_{e}\right]$ in the node $v$ an anomaly connected with the property $p$ occurred.

- $A C$ is an anomaly archive consisted of discovered anomalies that are sent from $A T$ after attack detection. However only anomalies connected with discovered attack are sent to the archive $A C$. In this way the time interval of discovered anomalies is not a criterion of sending given tuples $A\left(v, p, \alpha,\left[t_{b}, t_{e}\right]\right)$ into anomaly archive.

Anomaly table $A T$ is created for each monitoring region independently. On agents' autonomy assumption it is quite possible that one agent may discover anomaly state of the node $v$ with reference to some property's value, while other agent having the same node in its monitoring area will not observed any anomaly with reference to this property's value.

The third module closed in internal agents' knowledge structure is ontology module that consists of the attack patterns. Each monitoring agent subordinates only to one managing agent.

\subsection{The Characteristic of the Managing Agents}

Managing agents are responsible for coordination and management of the monitoring system. The internal structure of the managing agents is as follows. Each managing agent is equipped with: data storage module, data analysis module, attack detection module, queue task module and communication module. In the data storage module two types of data are stored: data received from the monitoring agents and knowledge about the pattern of attacks. Data sent by monitoring agents are derived from their anomaly detection modules. In the data analysis module conflicts of inconsistent knowledge incoming from distributed resources are solved applying consensus methods. Many a time managing agent after receiving the warn message from a one agent, asks other agents about their opinions. Also the new patterns of attacks are discovered in the data analysis module.

The attack detection module is responsible for the attack detections. In the attack detection module there are embodied two algorithms: the algorithm for determining of the sources attack and the algorithm for determining of the attack propagation scenarios. In this paper the attention to the first algorithm is paid, see Section 4 for details. The Queue task module consists of the tasks that must be executed or delegated by managing agent [17].

\section{The Language of Communication}

The agents communicate with each other to exchange knowledge about the states of monitored nodes or send the requests. They use a communication language compatible 
with standard ACL (Agent Communication Language) proposed by FIPA (Foundation of Intelligent Physical Agents) [15]. The ACL like KQML (Knowledge Query and Manipulation Language), developed by DARPA knowledge Sharing Effort, rely on speech act theory $[2,18]$. These languages base on the performatives. The messages might be sent to one or more receivers. From the number of receivers point of view two types of performatives are considered: peer-to-peer and multicast. In the algorithm for determining the set of Masters following types of messages are used: warn messages, multi_query_if messages, confirm (disconfirm) messages and multi_request performatives with inform action execution. A monitoring agent ma sends the warn message to the managing agent as a warning of the anomaly detection in his monitoring region. There must exist a tuple $A\left(v, p, \alpha,\left[t_{b}, t_{e}\right]\right)$ in the agent's ma anomaly table. The message with query_if performative is sent if the sender wants the receiver to ask about the anomaly appearance, i.e. the value of the parameter $p$ in a given node $v$ is higher than a given value $x$. Multi_query_if is used to send a query to a group of the agents. For example the managing agent may ask, if in the given time interval the value of the parameter $p$ in a node $v$ was abnormal i.e. higher than $x$ : multi_query_if $\left(A\left(v,(p>x), \alpha,\left[t_{b}, t_{e}\right]\right)\right)$.The confirm (disconfirm) message is send by the managing or monitoring agent if they want to confirm (disconfirm) the true of some proposition. If the managing agent sends confirm (disconfirm) message to more than only one agent then we have the multi_confirm (multi_disconfirm) message. The multi_request performative is sent by the managing agent to a group of monitoring agents. In this kind of message also the type of action must be specified. In the algorithm proposed in this paper an inform action is considered. In case of multi_request performatives with inform action execution the managing agent sends to the group of the monitoring agents a request of sending information about the discovered anomalies. If the managing agent requests of sending information about a state of a single node $v$ with the reference to the one parameter $p$ in the time interval $\left[t_{b}, t_{e}\right]$ then the message content is as follows: send_information $\left(O\left(v, p,\left[t_{b}, t_{e}\right]\right)\right)$. The managing agent may also ask about the nodes that have been communicated wit a given node. In this case the communication matrix is analyzed.

\section{The Algorithm for the DDoS Attack Detecting}

The algorithm for the sources of $D D o S$ attack detecting is embodied in the managing agent's attack detection module. It is assumed that network traffic should be analyzed in order to detect anomalies. The main purpose is to detect the attack before blocking the chosen goal. In order to detect the $D D o S$ attacks the anomalies in traffic characteristics and anomalies in communication schemes must be observed. In the DDoS attack it is assumed that Attacker is the source of attack and initializes the overall attack in a computer network by putting a maliciously software in chosen computers (nodes). These nodes become Masters. In the next step each Master starts to infect other nodes that become Demons. Next Demons infect other nodes and those next ones etc. and in this way spreading stage is expanded. When sufficient number of nodes is infected or 
at the fixed time point final attack or on earlier chosen computer (attack goal) is carried out. Due to the numerous numbers of requests sent simultaneously from different Demons at the same time interval to the attack goal all his resources are allocated and blocked [13].

\subsection{The Idea of the Algorithm}

The goal of applying the algorithm for the sources attack detecting is to determine the node $v^{k} \in V$ that is an Attacker. This algorithm consists of two main steps. In the first step the set of Masters is detected and in the second one on the base of Masters' input traffic analysis the Attacker is trailed. In this work the first Step is discussed in detail. The idea of the algorithm is as follows [17]:

Step 1. Determine the set $H$ of Masters.

Step 2. If the cardinality of the set $H$ is higher than threshold value $\tau$ then go to Step 3 else go to Step 1.

Step 3. Apply the procedure of the Attacker detection.

\subsection{The Algorithm for Determining the Set of the Masters}

Let us assume that the set of managing agents is one-element and we detect the anomaly with reference to one monitored property.

The algorithm for determining the set of the Masters is divided into two parts: Preliminary part and Principal part.

\section{Preliminary part}

Step 1. The monitoring agent $m a$ detects the anomaly in the node $v \in m r$ with the reference to the property $p \in P$. It means that there exists the tuple $\left(v, p, \alpha,\left[t_{b}, t_{e}\right]\right)$ in his anomaly table $A T$.

Step 2. The agent $m a$ determines the node $v_{k} \in m r$, in which the anomaly of the value of the parameter $p$ appeared at earliest.

Step 3. The monitoring agent $m a$ affirms the local anomaly in his monitoring region, indicates the node $v_{k}$ and sends a warn message to the managing agent.

Step 4. If the node $v_{k}$ is monitored by the other monitoring agents, then Go to the Step 5, else Go to the Step 8.

Step 5. The managing agent asks other monitoring agents about their opinions about the state of the node $v_{k}$ in a given time interval $\left[t_{b-\Delta}, t_{e}\right]$. The managing agent sends to them the message with multi_request performative with inform action execution.

Step 6. The managing agent collects the answers and obtains consistent opinion about the state of the node $v_{k}$. In this step, the procedure for verification of the node state is carried out, in which the consensus methods are applied. The aim of this procedure is to obtain the agreement of the node state.

Step 7. If anomaly exists then Go to Step 8 else send into the queue task module the task of the node state verification in some time.

Step 8. The managing agent initializes Principal part of the algorithm. 


\section{Principal part}

Step 1. The managing agent asks monitoring agents which nodes communicated with the node $v_{k}$ in the time interval $\left[t_{b-\Delta}, t_{b}\right]$. The managing agent sends the message with multi_request performative with inform action execution.

Step 2. Monitoring agents on the basis of their communication matrixes determine the nodes that communicated with the node $v_{k}$ in the time interval $\left[t_{b-\Delta}, t_{b}\right]$ and send the answers.

Step 3. The managing agent obtains the set $K$ of the nodes that communicated with the node $v_{k}$ in the time interval $\left[t_{b-\Delta}, t_{b}\right]$.

Step 4. The managing agent commissions the monitoring agents to verify if in the nodes from $K$ the anomaly of the value of the parameter $p$ in the time inter$\operatorname{val}\left[t_{b-\Delta}, t_{b}\right]$ appeared. For each $v \in K$ the managing agent sends the message with multi_guery_if performative.

Step 5. Monitoring agents on the basis of their anomaly tables send the answers to the managing agent.

Step 6. The managing agent determines the set of infected nodes $K$ '. If the nodes from $K^{\prime}$ are monitored by more than one monitoring agents, then the managing agent obtains the consensus opinion about the anomalies.

Step 7. If the set $K^{\prime}$ is not empty then go to Step 8 else go to Step 9.

Step 8. From the set $K^{\prime}$ managing agent selects the node $v^{\prime}$ that communicated with the node $v_{k}$ at earliest and applies for the node $v^{\prime}$ the Principal Part. Go to the Step 1.

Step 9. The node $v_{k}$ is a Master. Include $v_{k}$ into the set $H$. Stop.

\subsection{An Example}

Fig. 1 shows the anomaly of the input and output traffic in the node $v_{2} \in m r_{3}$. The node $v_{2}$ is monitored by the agent $m a_{3}$. This agent has observed the anomaly of the input traffic in the following time intervals: $\left[t_{3}, t_{7}\right],\left[t_{11}, t_{16}\right],\left[t_{18}, t_{20}\right]$ and $\left[t_{20}, t_{25}\right]$ with the reference to the values of $p_{1}$ and $p_{2}$. In the time intervals: $\left[t_{9}, t_{14}\right]$ and $\left[t_{19}, t_{23}\right]$ the anomaly of the output traffic has been observed. The nodes: $v_{3}, v_{13}, v_{17}, v_{16}, v_{15}$ communicated with $v_{2}$ while $v_{2}$ communicated with $v_{8}$ and $v_{19}$.

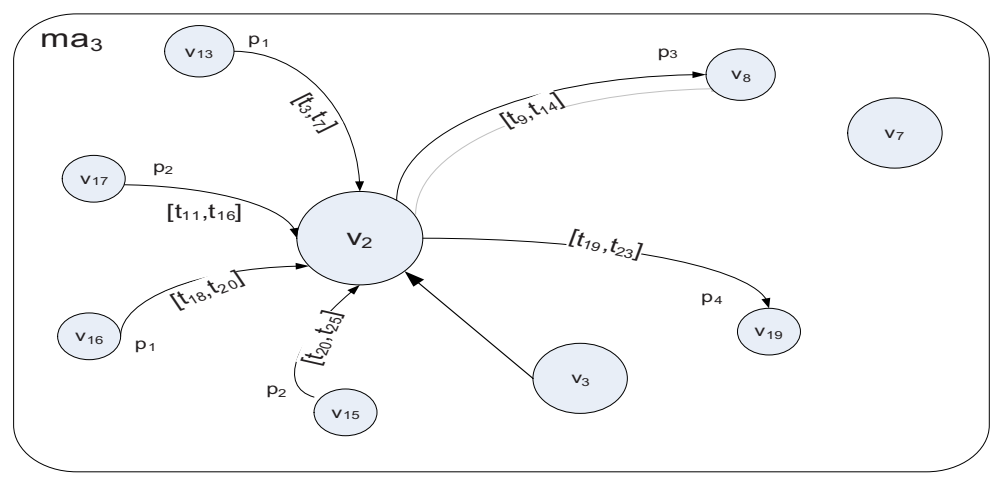

Fig. 1. Anomaly of the input and output traffic in the node $v_{2}$ 


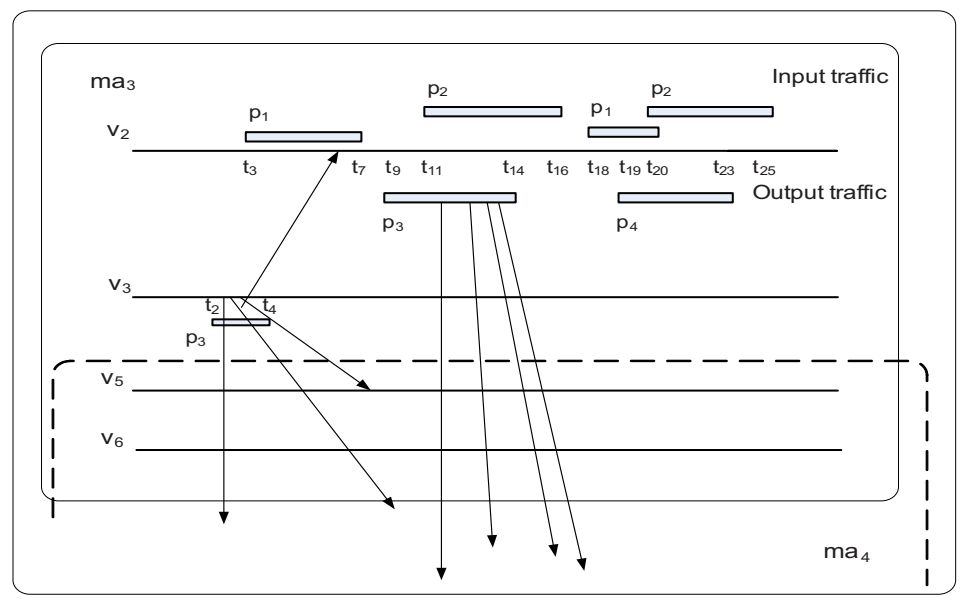

Fig. 2. Graphic representation of the piece of the anomaly table created by the $m a_{3}$.

- the anomaly of the parameter $p_{1}$ in the time intervals $\left[t_{3}, t_{7}\right]$ and $\left[t_{18}, t_{20}\right]$

- the anomaly of the parameter $p_{2}$ in the time intervals $\left[t_{11}, t_{16}\right]$ and $\left[t_{20}, t_{25}\right]$

- the anomaly of the parameter $p_{3}$ in the time interval $\left[t_{9}, t_{14}\right]$

- the anomaly of the parameter $p_{4}$ in the time interval $\left[t_{19}, t_{23}\right]$

Let us concentrate on the analyzing of the parameter $p_{3}$. On the basis of the time intervals in which this anomaly occurred we deduce that the node $v_{3}$ is the local source of the attack in the region $m r_{3}$. Let us assume the belief coefficient $\alpha$ equals 1 for all detected anomalies and the coefficient $\Delta$ equals 4 .

The algorithm for determining the set of the Masters is as follows.

\section{Preliminary part}

Step 1. The monitoring agent $m a_{3}$ detects the anomaly in the node $v_{2} \in m r_{3}$ with the reference to the property $p_{3} \in P$. It means that there exists the tuple $\left(v_{2}, p_{3}, 1,\left[t_{9}, t_{14}\right]\right)$ in his anomaly table $A T$.

Step 2. The agent $m a_{3}$ determines the node $v_{3} \in m r_{3}$, in which the anomaly of $p_{3}$ appeared at earliest.

Step 3. The monitoring agent $m a_{3}$ sends warn message to the managing agent.

Step 4 . The node $v_{3}$ is monitored only by the one agent.

Step 8. The managing agent initializes Principal part of the algorithm.

\section{Principal part}

Step 1. The managing agent asks monitoring agents which nodes communicated with the node $v_{3}$ in the time interval $\left[t_{0}, t_{4}\right]$. The managing agent sends the message with multi_request performative with inform action execution.

Step 2. Monitoring agents on the basis of their communication matrixes send the answers. 
Step 3. The managing agent receives the answers from two agents: $m a_{1}$ and $m a_{2}$ and determines the set $K=\left\{v_{7}, v_{9}, v_{12}\right\}$ (Fig 3).

Step 4. The managing agent commissions the monitoring agents to verify if in the nodes from $K$ the anomaly of the value of the parameter $p_{3}$ in the time inter$\operatorname{val}\left[t_{0}, t_{4}\right]$ appeared. The managing agent sends the message with multi_guery_if performative.

Step 5. Monitoring agents on the basis of their anomaly tables send the answers to the managing agent.

Step 6. The managing agent determines the set $K^{\prime}$ of infected nodes: $K=\left\{v_{7}, v_{12}\right\}$

Step 8. From the set $K^{\prime}$ managing agent selects the node $v_{7}$ and applies for the node $v_{7}$ the Principal Part. Go to the Step 1.

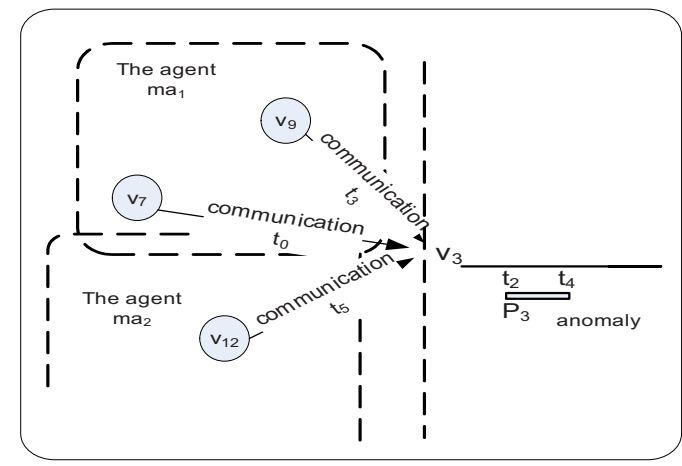

Fig. 3. The nodes $v_{7}, v_{9}$ and $v_{12}$ have communicated with the node $v_{3}$ and one of them has infected the node $v_{3}$.

\section{Conclusions}

In this paper the problem of automatic intrusion detection was discussed. This paper is a continuation of the issues presented in previous works $[11,14]$. In particular the ideas presented in [17] were here developed and the algorithm for automatic detecting of the DDoS attack was in detail described. This algorithm consists of two parts and the first part was here discussed. As a future work the second part of the algorithm must be developed and practical verification of presented results must be done.

Applying agency into the tasks of intrusions detection is the chance of building of the automatic intrusion detection systems that act in an incomplete, inconsistent and unpredictable environment. Agents as autonomous entities observe the nodes, take the decisions, communicate each other, integrate the results of their local activity and solve the global task of intrusion detection. In this paper we concentrate on the presentation of the algorithm for intrusion detection, and it was assumed that after discovering the anomaly of some parameter the agents apply the algorithm for the source of 
DDOS attack detection. It must be pointed out that the anomalies discovering plays a crucial role in a whole system. This problem basis not only on measuring some parameters and comparing them with templates but also requires taking into account other individual nodes' characteristics. Undoubtedly the effectiveness of monitoring system depends not only on the applied intrusion detection algorithms but also on the input of these algorithms i.e. effectiveness of lower layers responsible for anomalies detecting.

\section{References}

1. Ajith, A., Thomas, J., Han, S.Y.: D-SCIDS: Distributed soft computing intrusion detection system. Journal of Network and Computer Applications 30(1), 81-98 (2001)

2. Austin, J.L.: How to Do Things with Words, Cambridge (Mass.) (1962), Harvard University Press, 2nd edn. (2005)

3. Balasubramaniyan, J.S., Garcia-Fernandez, J.O., Isacoff, D., Spafford, E., Zamboni, D.: An Architecture for Intrusion Detection Using Autonomous Agents. In: Proceedings of the 14th Annual Computer Security Applications Conference (1998)

4. Biermann, E., Cloete, E., Venter, L.M.: A comparison of Intrusion Detection systems. Computers and Security 20(8), 676-683 (2001)

5. Bejtlich, R.: Tao of Network Security Monitoring. In: The: Beyond Intrusion Detection, Addison-Wesley, Reading (2004)

6. Crosbie, A.M., Spafford, G.: Applying Genetic Programming to Intrusion Detection. In: Proceedings of the AAAI Fall Symposium on Genetic Programming, AAAI Press, Cambridge, Menlo Park (1995)

7. Crosbie, B.M., Spafford, G.: Defending a Computer System using Autonomous Agents. In: Proceedings of the 18th National Information Systems Security Conference (1995)

8. Dasgupta, D.: Immunity-Based Intrusion Detection System: A General Framework. In: Proceedings of the 22nd National Information Systems Security Conference, USA (1999)

9. Denning, D.E., Edwards, D.L., Jagannathan, R., Lunt, T.F., Neumann, P.G.: A prototype IDES: A real-time intrusiondetection expert system. Technical report, Computer Science Laboratory, SRI International, Menlo Park (1987)

10. Fenet, S., Hassas, S.: A distributed Intrusion Detection and Response System based on mobile autonomous agents using social insects communication paradigm, Electronic Notes in Theoretical Computer Science (2001)

11. Kołaczek, G., Pieczyńska, A., Juszczyszyn, K., Grzech, A., Katarzyniak, R., Nguyen, N.T.: A mobile agent approach to intrusion detection in network systems. In: Khosla, R., Howlett, R.J., Jain, L.C. (eds.) KES 2005. LNCS (LNAI), vol. 3682, pp. 514-519. Springer, Heidelberg (2005)

12. KQML Specification, http://www.cs.umbc.edu/kqml/

13. Mirkovic, J., Dietrich, S., Dittrich, D., Reiher, P.: Internet Denial of Service: Attack and Defense Mechanisms. Prentice Hall, Englewood Cliffs (2005)

14. Nguyen, N.T., Juszczyszyn, K., Kołaczek, G., Grzech, A., Pieczyńska, A., Katarzyniak, R.: Agent-based approach for distributed intrusion detection system design. In: Alexandrov, V.N., van Albada, G.D., Sloot, P.M.A., Dongarra, J. (eds.) ICCS 2006. LNCS, vol. 3993, pp. 224-231. Springer, Heidelberg (2006)

15. FIPA Specifications, http://www. fipa.org/ 
16. Patcha, A., Park, J.-M.: An overview of anomaly detection techniques: Existing solutions and latest technological trends. Computer Networks 51(12), 3448-3470 (2007)

17. Prusiewicz (Pieczyńska) A.: A Multi-agent System for Computer Network Security Monitoring. In: KES-AMSTA 2008, LNCS (LNAI), vol. 4953, pp. 842-849 Springer, Heidelberg (2008)

18. Searle, J.: Speech Acts. Cambridge University Press, Cambridge (1969)

19. Spafford, E., Zamboni, D.: Intrusion detection using autonomous agents. Computer Networks: The International Journal of Computer and Telecommunications Networking 34(4), 547-570 (2000)

20. Wilson, E.: Network Monitoring and Analysis: A Protocol Approach to Troubleshooting. Prentice Hall, Englewood Cliffs (1999) 